\title{
Effect of Surface Treatment on Elemental Composition of Recast NiCr Alloy
}

\author{
Tevfik Yavuz ${ }^{1 *}$, Aslı Acar ${ }^{2}$, Serhan Akman ${ }^{1}$, Atiye Nilgun Ozturk ${ }^{1}$ \\ ${ }^{1}$ Department of Prosthodontics, Selcuk University, Konya, Turkey; ${ }^{2}$ Department of Prosthodontics, Gaziantep University, Gaziantep, \\ Turkey. \\ Email: *drtevfikyavuz@gmail.com
}

Received December $20^{\text {th }}, 2011$; revised January $27^{\text {th }}, 2012$; accepted February $26^{\text {th }}, 2012$

\begin{abstract}
Objective: The aim of this study was to compare the effects of different surface treatments on the elemental composition stability of as-received and recast types of $\mathrm{NiCr}$ casting alloys. Material and Methods: Six commercial NiCr dental casting alloys (Kera N, Nodelco, Bellabond, Wiron 99, Metaplus VK, and Tritech D) were used in this study. Seventy-two specimens $(12 \times 12 \times 1.2 \mathrm{~mm})$ were produced using the lost-wax casting process. Three casting protocols were established in relation to the proportion of as-received and recast alloys: Group A ( $100 \%$ as-received metal), Group B (50 wt \% new metal, $50 \mathrm{wt} \%$ once-recast metal), and Group C (100\% once-recast metal). Two specimens in every group received two different surface treatments: sandblasting with $110 \mu \mathrm{mm} \mathrm{Al}_{2} \mathrm{O}_{3}$ or oxidation firing. An elemental analysis was performed with x-ray energy-dispersive spectroscopy. Results were subjected to one-way analysis of variance and Tukey HSD tests. Results: The elemental composition was significantly different in the casting groups and treatment groups $(p<0.05)$. The lowest mean weight percentage Ni value was recorded for Group $\mathrm{C}$ and the highest for Group A. Aluminum-oxide sandblasting of the alloy surface reduced the mean weight percentage for Cr. Conclusion: Recasting metal alloys may adversely affect surface quality.
\end{abstract}

Keywords: Recasting; Elemental Composition; NiCr Alloy

\section{Introduction}

$\mathrm{Ni}-\mathrm{Cr}$ alloys possess good mechanical properties, such as high hardness, low density, and high tensile strength [1]. Besides the low cost and easy fabrication of $\mathrm{Ni}-\mathrm{Cr}$ alloys have made them widely used in the dental fixed prostheses for a long time [1]. For centuries, Ni-Cr has been used to fabricate fixed prostheses [2]. With the much less expensive $\mathrm{Co}-\mathrm{Cr}$ [3], Ni-Cr-Be [4], and $\mathrm{Ni}-\mathrm{Cr}$ [5] base metal alloys, remelting a small number of times does not appear to greatly affect their properties [6]. The effects of recasting on the mechanical properties of dental alloys and current issues relevant to these properties of recast alloys were presented [1]. Textbook guidelines for recasting Ni-Cr alloy vary from not adding any new metal, to some new metal, to $50 \%$ new metals with previously melted buttons or sprues removed from the castings [79]. The manufacturer's product information on $\mathrm{Ni}-\mathrm{Cr}$ casting alloys also typically states that scrap metals can be remelted to fabricate clinically acceptable castings, provided that at least $50 \%$ new metal is used. The basis for this empiric guideline is that certain important secondary elements, present in small percentages in the

*Corresponding author. original alloy compositions, may be lost during melting through volatilization or oxidation [2]. One example is zinc, which acts as an oxygen scavenger during melting to minimize the oxidation of other elements in the alloy [2].

Chromium is responsible for the tarnish and stainless properties of these alloys. When the chromium content of an alloy is over $30 \%$, the alloy is more difficult to cast. With this percentage of chromium, the alloy also forms a brittle phase, known as the sigma (6) phase [8]. Therefore, cast base metal dental alloys should not contain more than $28 \%$ or $29 \%$ chromium. In general, cobalt and nickel, up to certain percentage, are interchangeable elements [8].

Furthermore almost all elements in these alloys, such as chromium, silicon, molybdenum, and nickel, react with carbon to form carbids, which changes the properties of the alloys [8]. The presence of 3\% to $6 \%$ molybdenum contributes to the strength of the alloys [8].

Aluminum in nickel containing alloys forms a compound of nickel and aluminum $\left(\mathrm{Ni}_{3} \mathrm{Al}\right)$ [8]. This compound increases the ultimate tensile and yield strengths of the alloy considerably [8]. Silicon and manganese are 
added to increase the fluidity and castability of these alloys. There is a remarkable similarity in the properties of different alloys having a relatively wide variation in composition [8].

However, clinical practice shows the increased use of $\mathrm{Ni}-\mathrm{Cr}$ alloys for ceramometal restorations and the substitution of gold alloys [10]. In addition to the basic requirement of good resistance to change of chemical structure that is indispensable for any restorative metal material, Ni-Cr alloys also must have good castability, good interaction with dental ceramics, and ease of manipulation [10].

Repeated casting show more stability in nickel-based alloys. The problem associated with base metal alloys is that it is hard to cast them. Recasting of Ni-Cr alloys reveals no meaningful effects on their castability. But some studies, such asthose by Issac and Bath [11] andMirkovic [12], have demonstrated that recasting base metal alloys had negative effects on the alloys' properties and the cytotoxicity of base metal alloys increased after recasting [13].

The change in the values of Ni-Cr-Mo-Si recasting alloy process is important in this study. Also the contribution of the other elements thatexist in low ranges in alloys is important. It was determined how sandblasting and degassing affect the changes in the surface structure of alloys and elemental composition.

The studies onrecasting base metal alloys are restricted to examining the physical characteristics of these alloys [14]. The aim of this study was to compare the effects of different surface treatments on the elemental composition stability of as-received and recast type of $\mathrm{NiCr}$ casting alloys. The hypothesis for this study was that the recasting of base metal alloys would change the chemical properties of the alloys and thus affect the element release [15].

\section{Method and Materials}

Six Ni-Cr dental alloys-(KeraN, Eisenbacher Dentalwaren $\mathrm{GmbH}$, Woerth, Germany), (Nodelco, Kocaeli, Turkiye), (Bellabond, Bego, Bremen, Germany), (Wiron 99, Bego, Bremen, Germany), (Metaplus VK, AZ \& Partner AG, IPS d.SIGN; Ivoclar Vivadent), and Tritech D, Voerde, Germany) - were used in this study (Table
1).

\section{Preparation of Alloy Specimens}

The specimens $(10 \times 10 \times 1 \mathrm{~mm})$ from each type of alloy were prepared. Six groups of 72 specimens from each alloy type were fabricated as follows.

In the first group, the specimens were cast new alloys. The second group consisted of specimens that were cast from $50 \mathrm{wt} \%$ new alloys with $50 \mathrm{wt} \%$ recast (once) alloys. The third group consisted of specimens that were cast from $100 \%$ recast (once) alloys. The metal used for recasting was obtained from leftover buttons of the alloys that had been previously cast.

The specimens were fabricated using a conventional lost-wax technique. For the preparation of metal specimens, threegroups of 24 wax patterns each $(10 \times 10 \times 1$ $\mathrm{mm}$ ) were fabricated. The wax patterns were invested using a phosphate bond investment (Bellawest, Bego, Bremen, Germany) and submitted to the lost-wax casting process on a gas/oxygen flame and then to centrifugation. The rings were submitted to thermal cycling according to the manufacturers' recommendations, and a casting temperature of $1330^{\circ} \mathrm{C}$ was used. After the sprues had been cut off, the specimens were airborne-particle abraded using aluminum oxide (Alox, Bego, Bremen, Germany) in order to be cleaned from investment material. Then specimens were polished and finished by stone burs (Vlachovicka 619, Moravia, Nove 59231, Czech Republic) and rubber polishing wheels (BEGO). The specimens were then placed in sterile distilled water and cleaned by sonication (BioSonic Jr., Whaledent, New Jersey, USA).

The specimens were divided randomly into two groups for sandblasting and degassing. The $\mathrm{Ni}-\mathrm{Cr}$ casting specimens, with a dimension of $(10 \times 10 \times 1 \mathrm{~mm})$ were sandblasted and steam-cleansed, as described above, and then underwent $970^{\circ} \mathrm{C}$ oxidizing heat treatment for $10 \mathrm{~min}$ (Traditionally and imperfectly termed degassing).

The specimens' compositions in weight percentages were calculated with the Energy Dispersive X-Ray Analysor System (EDX) (Tracar Northern TN-5500 Energy Dispersive System).

Means and standard deviations were calculated for each group, and the results were analyzed with one-way ANOVA and Tukey HSD tests. Statistical differences

Table 1. Dental cast alloys used.

\begin{tabular}{|c|c|c|}
\hline Alloy & Type of alloy & Composition (wt\%) \\
\hline Kera N & Ni-Cr: Base metal & Ni: 62, Cr: 25, Mo: 10.62, Si: 1.4, Mn: 0.01, C: 0.02 \\
\hline Bellabond & Ni-Cr: Base metal & Ni: 65.2, Cr: 22.5, Mo: 9.5, (Fe, Si, Mn, Nb) $<2$ \\
\hline Nodelco & Ni-Cr: Base metal & Ni: 62, Cr: 25, Mo: 9.5, Si: 0.99, C: 0.5, Nb: 1 \\
\hline Wiron 99 & Ni-Cr: Base metal & Ni: 65, Cr: 22.5, Mo: 9.5, (Si, Nb, Fe, Ce $)<1$ \\
\hline Metaplus VK & Ni-Cr: Base metal & Ni: 61.2, Cr: 25.8, Mo: $11 \mathrm{Si}: 1.5(\mathrm{Al}, \mathrm{Mn})<1$ \\
\hline Tritech D & Ni-Cr: Base metal & Ni: 64, Cr: 24.5, Mo: 10, Si: 1.2, Nb: $0.3, \mathrm{Ce}: 0.3$ \\
\hline
\end{tabular}


were determined at the $95 \%$ confidence level $(p=0.05)$ All statistical analyses were performed using the Statistical Package for Social Sciences, vers.13.0 (SPSS, Chicago, IL).

\section{Results}

One-way ANOVA showed that both sandblasting and degassing procedures had a significant effect $(p<0.05)$. Further comparison with Tukey's analyses showed that alloys cast from $100 \%$ new materials showed significant differences in chemical structure in comparison to oncerecast, $50 \%$ new, and $50 \%$ once-recast groups $(p<0.05)$. The chemical structure for each alloy wascompared. Overall, there was a significant reduction in the Ni ratio in the degassing group comparison to the sandblasting group (Figure 1) $(p<0.05)$. There was significant increase ratio of $\mathrm{Cr}$ for the degassing group in comparison to the sandblasting group for all the alloys $(p<0.05)$.

\section{Discussion}

Since 1962, studies regarding recasting of base metal alloys have been conducted by various researchers, namely Harcourt [16], Hesby [3], Hong [17], Nelson [18], and Presswood $[4,19]$. They mainly studied properties of recast alloy like tensile strength, ultimate tensile strength, percentage elongation, modulus of elasticity, mean yield strength, microstructure, and micro hardness. The findings of the current study agreed with the hypothesis that recasting of base metal alloys would change the chemical structures and elemental compositions. The fabrication of the alloy specimens was performed in a way to simulate the technique used to produce dental cast restorations in dental laboratories [15]. Finally, although a small sample size $(n=6)$ was used in the current study, significant differences were found between the different groups, indicating a sufficiently large effect size [15].

Preswood [4] has analyzed the chemical composition of $\mathrm{Ni}-\mathrm{Cr}-\mathrm{Be}$ that was recast fivetimes without adding

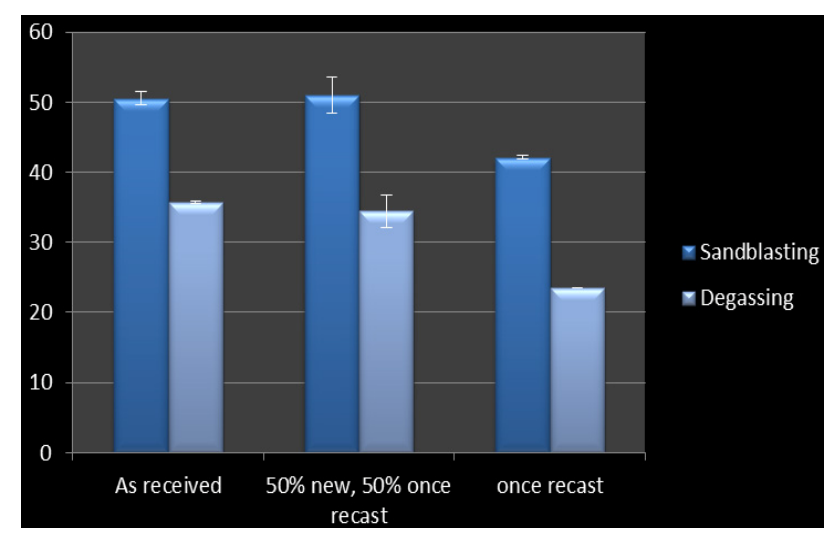

Figure 1. Example for Ni (Bellabond). new alloy by AAs. He indicated the changes in the amount of $\mathrm{Ni}, \mathrm{Fe}, \mathrm{Mn}, \mathrm{Cr}$, Mo, and $\mathrm{Al}$ remained within $0.1 \%-0.01 \%$ after each smelting and hence this alloy could be cast sixtimes without any significant changes in its chemical structure [4].

Nelson et al., [18] demonstrated no remarkable degenerative change in $\mathrm{Ni}-\mathrm{Cr}$ alloy recasting for 10 generations and combining used metal with new metal and recasting 100 times demonstrated no remarkable degenerative changes in physical properties, microstructure, or clinical characteristics, but strict adherence to clean techniques was essential to minimize contamination and inclusions that adversely affect the physical properties [20].

Beside the Ni-Cr elements, Mo is third most important element in Ni-Cr alloys. Mo is a refractory metal and not readily oxidized, but the oxide product of Mo (e.g., $\mathrm{MoO}_{3}$ ) becomes volatileat temperatures exceeding $700^{\circ} \mathrm{C}$ [1]. All six different brands of Ni-Cr alloys used in this research contained Mo in the bulk alloy [1]. The Mo content on the surface oxide layers for these $\mathrm{Ni}-\mathrm{Cr}$ alloys after heat treatment at $990^{\circ} \mathrm{C}$ for 10 min was almost negligible [1]. This phenomenon was believed to be ascribed to the volatility of Mooxides (mainly as $\mathrm{MoO}_{3}$ ) during heat treatment prior to firing of the opaque porcelain [1].

Phillips [7] found that the elemental composition of a $\mathrm{Ni}-\mathrm{Cr}$ alloy changed during melting procedures. The results of this study also indicated that mixing new and previously cast metal influenced the compositional stability of a Ni-Cr base metal alloy, leading to changes in the amount of base metals ( $\mathrm{Ni}$ and $\mathrm{Cr}$ ) and other major and minor elements (Mo, Si, and $\mathrm{Mn}$, etc.). In this study, changes in elemental concentrations were generally minimal, given that the practical accuracy of values determined by EDAX [2]. A nonhomogeneous distribution of alloy elements along the specimen surface may have influenced the results [2].

Sandblasting also led to a drop in the wt $\%$ concentration of a Ni-Cr and other elements. This significant change of the alloy surface composition after sandblasting might offer a possible explanation for the difference in bond strength values [21].

As a result, the common dental laboratory practice of repeatedly reusing casting alloys with random amounts of new metal additions is contraindicated [2]. Good restoration requires certain optimum properties. These properties should remain constant not only during various laboratory procedures but also in oral environment. Therefore, it is very clear that recasting should not be done at the expense of the properties of the alloy [19]. However, the small decrease in the concentration of Ni probably would have no clinically significant effect on the mechanical properties or corrosion resistance of the cast alloys [2]. To monitor the reuse of alloys, laboratories should institute effective approaches to metal handling 
and accounting [2]. Further means to facilitate reclamation of the used alloy in an efficient, expeditious, and economic manner by the manufacturer would encourage laboratories to return the large, unmanageable buttons that tend to accumulate [2].

With regard to the repeated process, it has been stated that the compounds of the oxidize layer and the changes in its thickness affect the porcelain linkage [17,18,22, 23].

In summary, reuse of the alloys at $50 \%$ and $100 \%$ as recast alloys significantly changed the elemental composition of the base metal alloys investigated in this study.

\section{Conclusion}

The recurrent casting of alloys may cause surface impairment and compositional deviations in alloys that may further induce organic hazards during their clinical uses. The very low change ratio of the elements affected the physical and biological properties of the alloy. In the research studies concerning the subject, it has been expressed that the repeated process causes microstructural changes like reduction in the elements $\mathrm{Cu}, \mathrm{Sn}, \mathrm{Zn}, \mathrm{Cr}$ and $\mathrm{Ti}$, larger particle size, dirtiness and microporosities resulting in increased casting defects which means badly affected mechanical properties. This type of process also causes reduction in the elements $\mathrm{Cu}, \mathrm{Sn}, \mathrm{Zn}$ and $\mathrm{Cr}$ which play role in the oxide layer production, larger porosities in quality-quantity and increased oxide layer thickness. These changes may have effect on the bond strength.

\section{REFERENCES}

[1] H. H. Huang, M. C. Lin, T. H. Lee, H. W. Yang, F. L. Chen, S. C. Wu and C. C. Hsu, "Effect of Chemical Composition of Ni-Cr Dental Casting Alloys on the Bonding Characterization between Porcelain and Metal," Journal of Oral Rehabilitation, Vol. 32, No. 3, 2005, pp. 206-212. doi:10.1111/j.1365-2842.2004.01411.x

[2] M. F. Ayad, "Compositional Stability and Marginal Accuracy of Complete Cast Crowns Made with As-Received and Recast Type III Gold Alloy," Journal of Prosthetic Dentistry, Vol. 87, No. 2, 2002, pp. 162-166. doi: $10.1067 / \mathrm{mpr} .2002 .121024$

[3] D. A. Hesby, P. Kobes, D. G. Garver and G. B. Pelleu Jr., "Physical Properties of a Repeatedly Used Nonprecious Metal Alloy," Journal of Prosthetic Dentistry, Vol. 44, No. 3, 1980, pp. 291-293. doi:10.1016/0022-3913(80)90014-1

[4] R. G. Presswood, "Multiple Recast of a Nickel-ChromiumBeryllium Alloy," Journal of Prosthetic Dentistry, Vol. 50, No. 2, 1983, pp. 198-199. doi:10.1016/0022-3913(83)90012-4

[5] S. Ozdemir and A. Arikan, "Effects of Recasting on the Amount of Corrosion Products Released from Two Ni-Cr Base Metal Alloys," European Journal of Prosthodontics and Restorative Dentistry, Vol. 6, No. 4, 1998, pp. 149153.

[6] R. H. Liu, W. M. Johnston, J. A. Holloway, W. A. Brantley and T. Dasgupta, "The Effect of Metal Recasting on Porcelain-Metal Bonding: A Force-to-Failure Study," Journal of Prosthetic Dentistry, Vol. 104, No. 3, 2010, pp. 165-172. doi:10.1016/S0022-3913(10)60114-X

[7] K. J. Anusavice, "Phillips' Science of Dental Materials," 10th Edition, WB Saunders, Philadelphia, 1996.

[8] R. G. Craig, J. M. Powers and J. C. Wataha, "Dental Materials Properties and Manipulation," 11th Edition, C. V. Mosby Company, St. Louis, 2001.

[9] L. Baum, R. W. Phillips and M. R. Lund, "Textbook of Operative Dentistry," 3rd Edition, WB Saunders, Philadelphia, 1995.

[10] O. L. Bezzon, R. F. Ribeiro, J. M. Rollo and S. Crosara, "Castability and Resistance of Ceramometal Bonding in Ni-Cr and Ni-Cr-Be Alloys," Journal of Prosthetic Dentistry, Vol. 85, No. 3, 2001, pp. 299-304. doi: $10.1067 / \mathrm{mpr} .2001 .113779$

[11] L. Issac and S. Bhat, "Effect of Re-Using NickelChromium Alloy on Its Ultimate Tensile Strength, Yield Strength and Modulus of Elasticity," Indian Journal of Dental Research, Vol. 9, No. 1, 1998, pp. 13-17.

[12] N. Mirkovic, "Effect of Recasting on the Elastic Modulus of Metal-Ceramic Systems from Nickel-Chromium and Cobalt-Chromium Alloys," Vojnosanitetski Pregled, Vol. 64, No. 7, 2007, pp. 469-473. doi:10.2298/VSP0707469M

[13] A. S. Al-Hiyasat, O. M. Bashabsheh and H. Darmani, "An Investigation of the Cytotoxic Effects of Dental Casting Alloys," International Journal of Prosthodontics, Vol. 16, No. 1, 2003, pp. 8-12.

[14] M. R. Nakhaei, J. Ghanbarzadeh and R. Goharian, "The Effect of Recast Base Metal Alloys on Crown's Marginal Accuracy," Journal of Medical Sciences, 2008, Vol. 8, No. 6, pp. 599-602. doi:10.3923/jms.2008.599.602

[15] A. S. Al-Hiyasat and H. Darmani, "The Effects of Recasting on the Cytotoxicity of Base Metal Alloys," Journal of Prosthetic Dentistry, Vol. 93, No. 2, 2005, pp. 158-163. doi:10.1016/j.prosdent.2004.11.009

[16] H. J. Harcourt and W. F. Cotterill, "Induction Melting of Cobalt-Chromium Alloys; a Comparison with Flame Melting," British Dental Journal, Vol. 20, No. 118, 1965, pp. 323-329.

[17] J. M. Hong, M. E. Razzoog and B. R. Lang, "The Effect of Recasting on the Oxidation Layer of a Palladium Silver Porcelain Alloy," Journal of Prosthetic Dentistry, Vol. 59, No. 4, 1988, pp. 420-425. doi:10.1016/0022-3913(88)90035-2

[18] D. R. Nelson, J. F. Palik, H. F. Morris and M. C. Comella, "Recasting a Nickel-Chromium Alloy," Journal of Prosthetic Dentistry, Vol. 55, No. 1, 1986, pp. 122-127. doi:10.1016/0022-3913(86)90087-9

[19] Jayant Palaskar, D. V. Nadgir and I. Shah, "Effect of Recasting of Nickel-Chromium Alloy on Its Hardness," International J of Dental Clinics, Vol. 2, No. 4, 2010, pp. 8-11. 
[20] A. A. Al-Ali, "Evaluation of Macrohardness of Recasted Cobalt-Chromium Alloy," Al-Rafidain Dental Journal, Vol. 7, No. 1, 2007, pp. 111-117.

[21] H. Petridis, P. Garefis, H. Hirayama, N. M. Kafantaris and P. T. Koidis, "Bonding İndirect Resin Composites to Metal: Part 2. Effect of Alloy Surface Treatment on Elemental Composition of Alloy And Bond Strength," International Journal of Prosthodontics, Vol. 17, No. 1, 2004, pp. 77-82.
[22] E. Papazoglou, W. A. Brantley, W. M. Johnston and A. B. Carr, "Effects of Dental Laboratory Processing Variables and in Vitro Testing Medium on the Porcelain Adherence of High-Palladium Casting Alloys," Journal of Prosthetic Dentistry, Vol. 79, No. 5, 1998, pp. 514-519. doi:10.1016/S0022-3913(98)70171-4

[23] M. Gladwin and M. Bagby, "Clinical Aspects of Dental Materials," Lippincott Williams \& Wilkins, Philadelphia 2000 . 